\title{
Fresnel Lens with Embedded Vortices
}

\author{
Sunil Vyas, ${ }^{1}$ Rakesh Kumar Singh,, ${ }^{1}$ Devinder Pal Ghai, ${ }^{2}$ \\ and P. Senthilkumaran ${ }^{1}$ \\ ${ }^{1}$ Department of Physics, Indian Institute of Technology Delhi, New Delhi 110016, India \\ ${ }^{2}$ Laser Science and Technology Centre, Metcalfe House, Delhi 110054, India
}

Correspondence should be addressed to P. Senthilkumaran, psenthilk@yahoo.com

Received 25 March 2011; Accepted 20 June 2011

Academic Editor: Jan Masajada

Copyright (c) 2012 Sunil Vyas et al. This is an open access article distributed under the Creative Commons Attribution License, which permits unrestricted use, distribution, and reproduction in any medium, provided the original work is properly cited.

\begin{abstract}
Vortices of different charges are embedded in a wavefront that has quadratic phase variation, and the intensity distribution near the focal plane is studied. This method may be useful in realizing complicated beam profiles. We have experimentally demonstrated the generation of vortex arrays having integer as well as fractional topological charges that produce different intensity profiles at the focal plane. The phase variation realized on a spatial light modulator (SLM) acts as a Fresnel lens with embedded vortices.
\end{abstract}

\section{Introduction}

A modulo $2 \pi$ operation carried out on a spherical wavefront results in concentric zones called Fresnel zones. A Fresnel lens is formed by segmenting the continuous surface of a conventional lens into a set of surfaces of the same curvature. This process allows fabrication of a large diameter lens with very short focal length thereby ensuring substantial increase in its numerical aperture. Further, there is a significant reduction in the thickness and weight of the lens. Thus, a plane wave incident on a Fresnel lens is split into multiple segments (zones), and the combination of multiple successive wavefront zones reconstructs the spherical wavefront.

An optical beam with phase singularity also called optical vortex or a screw dislocation is a helical phase ramp which focuses to a ring-like intensity pattern [1]. An optical vortex is characterized by azimuthal phase dependence of the type $\exp (\operatorname{im} \theta)$ where $m$ is the topological charge. The magnitude of topological charge represents the total phase accumulated by the helical wave in one complete revolution around the vortex point. The sign of topological charge represents left- or right-handed helicity. When the topological charge has a noninteger value, it results in the formation of a fractional vortex. Wavefront containing fractional charge vortex exhibits a half-cut (low-intensity radial opening in the doughnut structure), corresponding to a mixed edge-screw dislocation [2]. Such vortices, on propagation, transform into several opposite-sign screw dislocations which disappear on the beam periphery [3].

Optical vortices find applications in particle trapping [4], data encryption [5], phase contrast microscopy [6], image processing [7], collimation testing [8], vortex coronagraphy, [9] and in various other areas. Recently, optical vortex arrays which are regular nets of vortices have generated an enormous interest among many research groups [10, 11]. Optical vortex arrays, also called vortex lattices, find applications in multiple trap optical tweezers [12], spatial solitons [13], microfluidic sorting [14], metrology [15], micromechanics [16], and atom optics [17]. Vortex arrays are generated using interferometry [18-20], Talbot imaging [21], and spatial filtering [22]. In this paper, we present a simple and effective method of generating array of vortices by embedding vortex phase distribution in the Fresnel lens. Specially designed phase masks are produced which are encoded onto the SLM to achieve array of vortices near the focal plane. A vortex array of integer as well as fractional topological charge can be produced by this method. As this method uses a single lens function, there is no cross-talk and overlapping of different beams. The method offers flexibility in the selection of location, number density, and topological charge of vortices in the beam. 


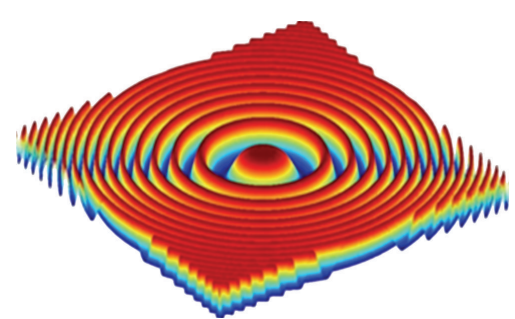

(a)

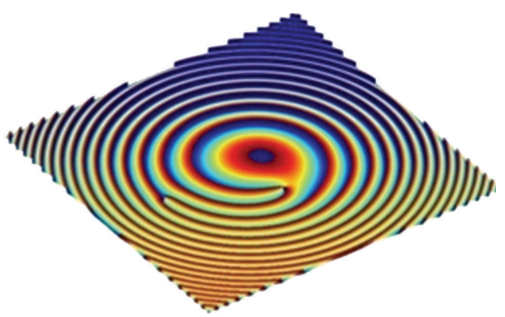

(c)

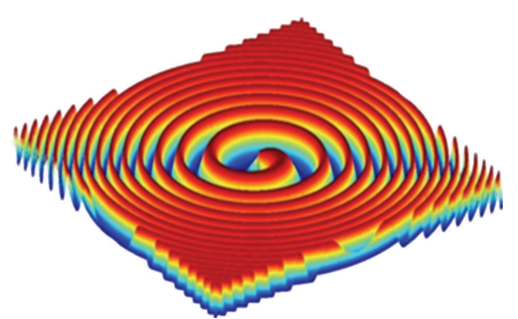

(b)

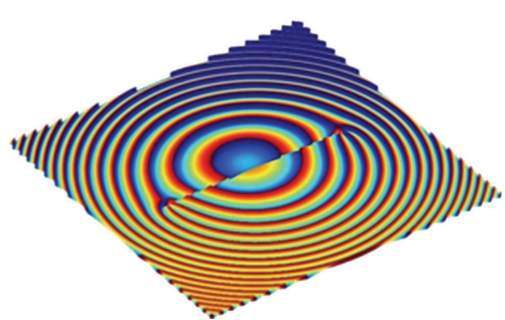

(d)

Figure 1: (a) Surface profile of a conventional Fresnel lens. (b) Surface profile of a Fresnel vortex lens. (c) Surface profile of a Fresnel lens with vortices embedded at off-axis locations. (d) Surface profile of a Fresnel lens with half-integer vortices embedded at off-axis locations.

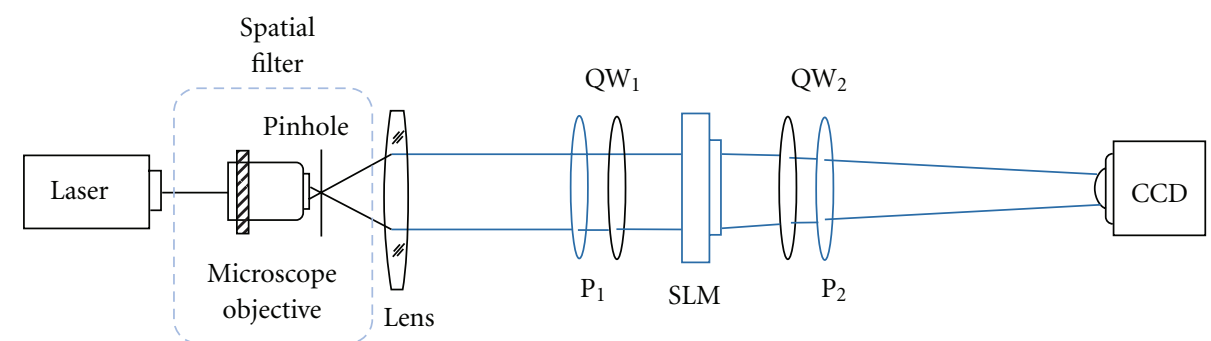

Figure 2: Experimental setup of Fresnel lens with embedded vortices; $\mathrm{P}_{1}$ and $\mathrm{P}_{2}$ : Polarizers, $\mathrm{QW}_{1}$ and $\mathrm{QW}_{2}$ : Quarter wave plates, SLM: Spatial light modulator, and CCD: Camera.

\section{Theory}

A convex lens brings a collimated incident beam to a focus at a distance $f$ from the lens. The phase delay $\Delta \psi(\rho, \theta)$ suffered by the beam due to lens is

$$
\Delta \psi(\rho, \theta)=\frac{-k \rho^{2}}{2 f}
$$

where $f$ is the focal length, $k=2 \pi / \lambda$, and $\lambda$ is the wavelength of light used. The polar coordinates in the element plane $\rho, \theta$ are related to Cartesian coordinates by $\rho^{2}=x^{2}+y^{2}, \theta=$ $\tan ^{-1}(y / x)$. A thin lens can be considered equivalent to a transparency with a complex amplitude transmittance given by

$$
t_{1}(x, y)=\exp (i \Delta \psi(\rho, \theta))
$$

Fresnel zone sectioning of this transmittance function leads to a Fresnel lens. The radius of the nth zone is $\rho_{n} \approx \sqrt{2 f n \lambda}$. Figure 1(a) shows the profile of a conventional Fresnel lens.
A vortex or phase singularity in the wave field can be implanted by using a phase mask or by a spiral phase plate. The transmission function of such a phase mask is given by

$$
t_{2}(\rho, \theta)= \begin{cases}\exp (i m \theta), & \text { for } 0 \leq \rho \leq 1 \\ 0, & \text { otherwise }\end{cases}
$$

where $m$ is the topological charge of vortex. A vortex lens [23] has the transmission function given by

$$
t_{3}(\rho, \theta)=t_{1}(\rho, \theta) t_{2}(\rho, \theta)=\exp \left(i\left(m \theta-\frac{k}{2 f} \rho^{2}\right)\right) .
$$

A phase mask having this transmittance function transforms incident plane wave into converging helicoidal wave $E(\rho, \theta, z)$. Presence of Fresnel lens factor in a wave field $e^{i m \theta}$ gives desired curvature to the beam. When the topological charge $m$ is a fraction, it is called fractional vortex lens [24]. This specific transmittance imparts an on-axis vortex on the incident optical field. Phase variation corresponding to 


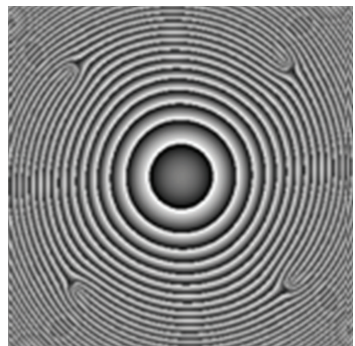

(a)

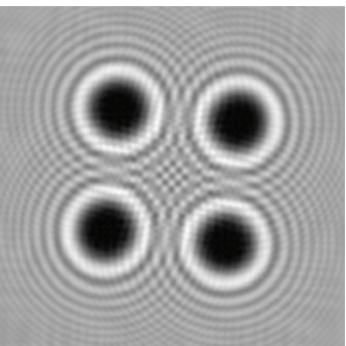

(b)

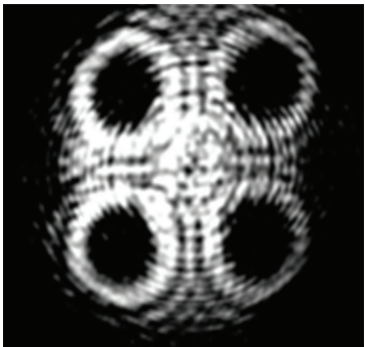

(c)

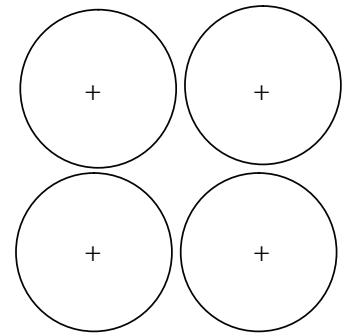

(d)

FIgURe 3: Fresnel lens with four vortices, each of topological charge 2 embedded at off-axis locations. (a) Phase distribution displayed on SLM. (b) Simulated intensity distribution near the focal plane. (c) Experimentally observed intensity distribution near the focal plane. (d) Distribution of topological charge in the intensity pattern.

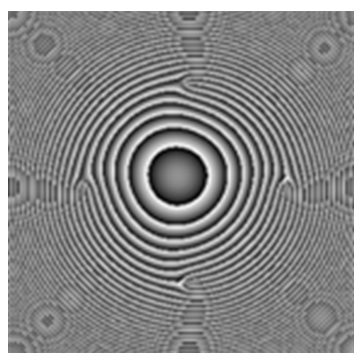

(a)

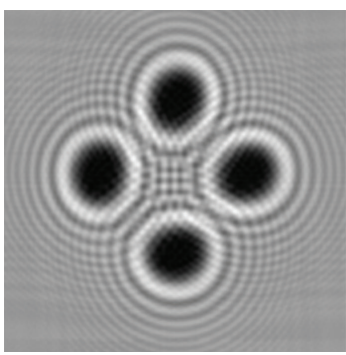

(b)

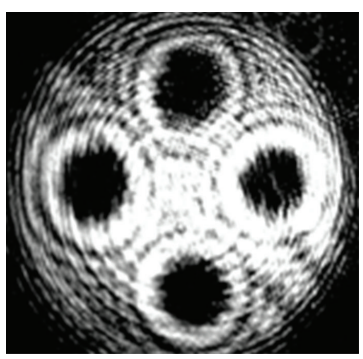

(c)

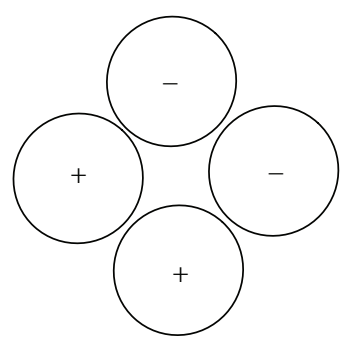

(d)

FIGURE 4: Fresnel lens with two positive and two negative charge vortices embedded at off-axis locations. (a) Phase distribution displayed on SLM. (b) Simulated intensity distribution near the focal plane. (c) Experimentally observed intensity distribution near the focal plane. (d) Distribution of topological charge in the intensity pattern.

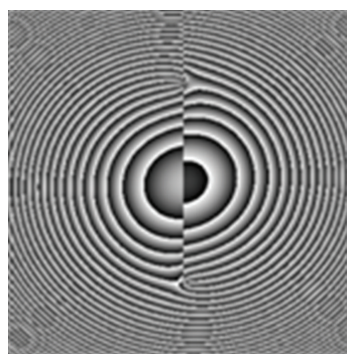

(a)

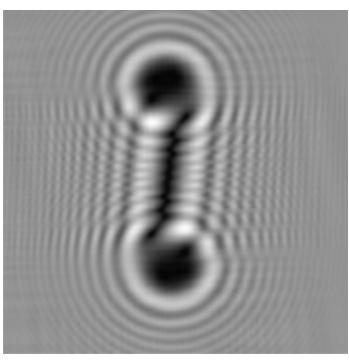

(b)

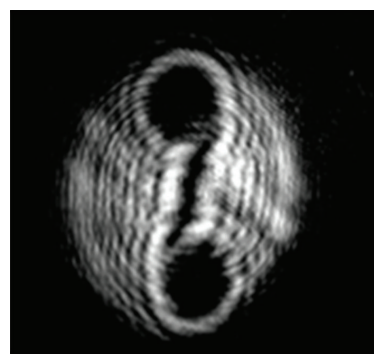

(c)

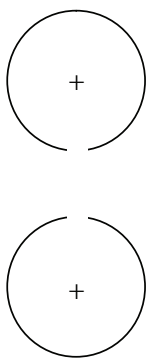

(d)

FIgURE 5: Fresnel lens with fractional vortices of the same charge (3.5) embedded at off-axis locations. (a) Phase distribution displayed on SLM. (b) Simulated intensity distribution near the focal plane. (c) Experimentally observed intensity distribution near the focal plane. (d) Distribution of topological charge in the intensity pattern.

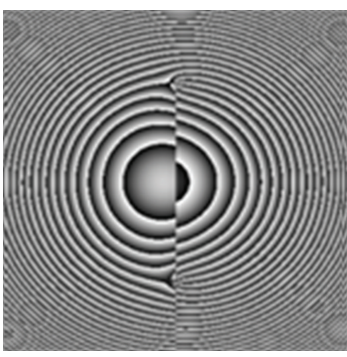

(a)

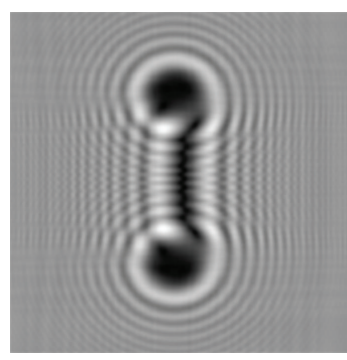

(b)

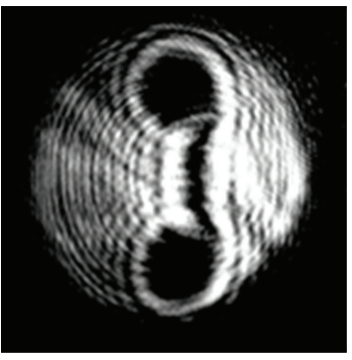

(c)
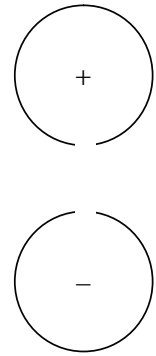

(d)

Figure 6: Fresnel lens with fractional vortices of opposite charge (3.5) embedded at off-axis locations. (a) Phase distribution displayed on SLM. (b) Simulated intensity distribution near the focal plane. (c) Experimentally observed intensity distribution near the focal plane. (d) Distribution of topological charge in the intensity pattern. 


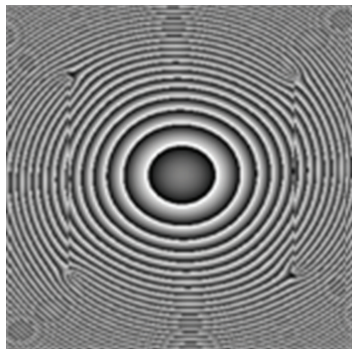

(a)

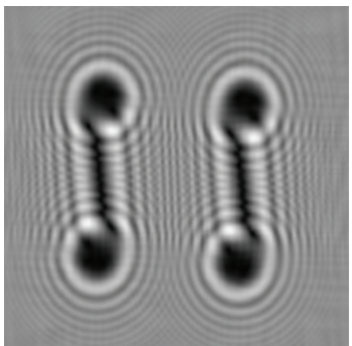

(b)

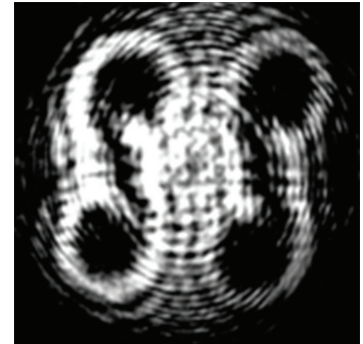

(c)

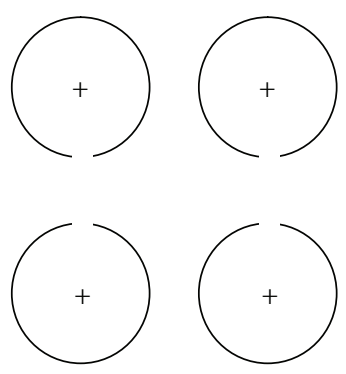

(d)

Figure 7: Fresnel lens with $2 \times 2$ lattice of fractional vortices of the same charge $m=3.5$ embedded at off-axis locations. (a) Phase distribution displayed on SLM. (b) Simulated intensity pattern near the focal plane. (c) Experimentally observed intensity distribution near the focal plane. (d) Distribution of topological charge in the intensity pattern.

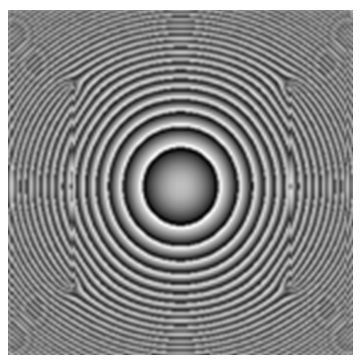

(a)

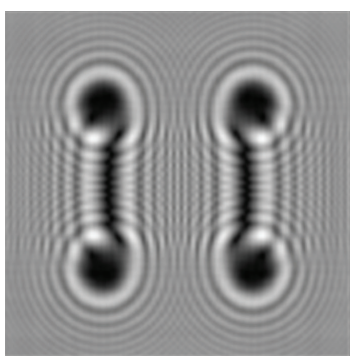

(b)

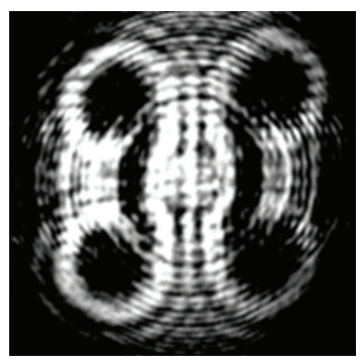

(c)

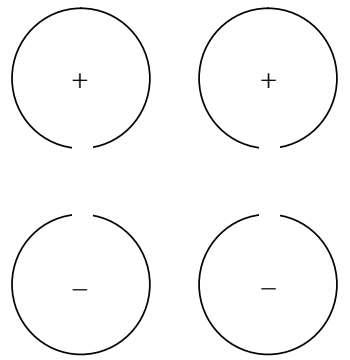

(d)

Figure 8: Fresnel lens with $2 \times 2$ lattice of fractional vortices with two positive and two negative topological charge vortices, each of magnitude 3.5 embedded at off-axis locations. (a) Phase distribution displayed on SLM. (b) Simulated intensity pattern near the focal plane. (c) Experimentally observed intensity distribution near the focal plane. (d) Distribution of topological charge in the intensity pattern.

multiple vortices can be embedded into the wavefront by using the expression

$$
t_{4}(\rho, \theta)=\exp \left[i \sum_{j=1}^{N} m_{j} \theta\left(\rho_{j}, \theta_{j}\right)\right],
$$

where $m_{j}$ is the topological charge of $j$ th vortex, and $\theta$ is helical phase variation corresponding to the $j$ th vortex. The position of the core of the $j$ th vortex is $\left(\rho_{j}, \theta_{j}\right)$, where

$$
\begin{gathered}
\rho_{j}=\sqrt{x_{j}^{2}+y_{j}^{2}}, \\
\theta_{j}=\tan ^{-1}\left(\frac{y-y_{j}}{x-x_{j}}\right),
\end{gathered}
$$

where $x_{j}, y_{j}$ are the Cartesian coordinates of the $j$ th vortex core. Similar method of embedding vortices at desired locations is used in the iterative computational phase synthesis algorithms for diffractive elements, in wave optical engineering $[25,26]$. This process is used to annihilate stagnation causing vortices, in the iterative algorithms.

When the extremum of a quadratic phase variation of a lens $\Delta \psi(\rho, \theta)$ coincides with the position of the core of the vortex, spiral zones are formed. Figure 1(b) shows the profile of a vortex Fresnel lens. When the position of the vortex $\left(\rho_{j}, \theta_{j}\right)$ does not coincide with the extremum of
$\Delta \psi(\rho, \theta)$, branching of the zone boundary occurs as can be seen from Figure 1(c). The number of branches is equal to the magnitude of the charge of the vortex. The sign of the vortex decides the sense (clockwise or anticlockwise) in which the branching occurs. Figure 1(d) shows profile of a vortex Fresnel lens in which opposite vortices of unequal fractional charges are embedded at two different locations.

The complex amplitude at the focal plane can be evaluated by using the Fresnel-Kirchhoff diffraction integral which can be written [27] as

$$
\begin{aligned}
E(r, \phi, f)= & A \int_{0}^{1} \int_{0}^{2 \pi} E(\rho, \theta, z=0) \\
& \times \exp \left[-i \frac{2 \pi}{\lambda f} r \rho \cos (\theta-\phi)\right] \rho d \rho d \theta
\end{aligned}
$$

where

$$
A=\frac{1}{i \lambda f} e^{i k f} \exp \left(\frac{i \pi r^{2}}{\lambda f}\right) .
$$

$(\rho, \theta, z)$ and $(r, \phi, f)$ correspond to object and diffracted field planes expressed in cylindrical coordinates. $\lambda$ is the wavelength of light used, and $f$ is the focal length of the lens. The multiplicative factor $A$ is ignored since the amplitude $1 / \lambda f$ is only a scale factor, and the phase $\left(k f+\left(\pi r^{2} / \lambda f-\pi / 2\right)\right)$ 


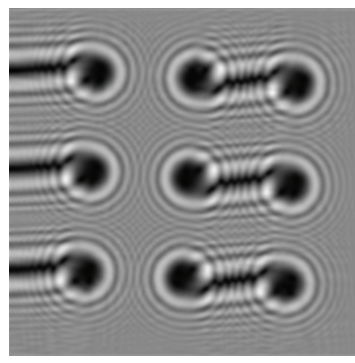

(a)

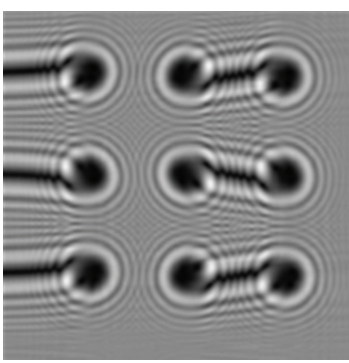

(b)

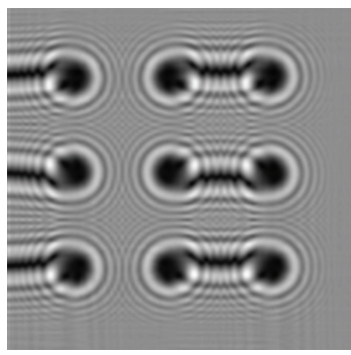

(c)

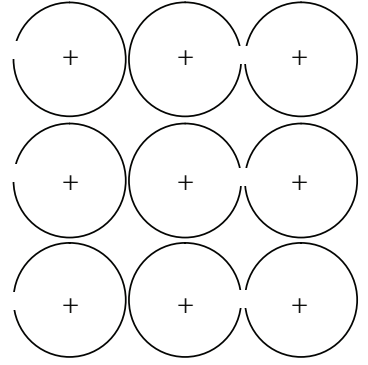

(d)

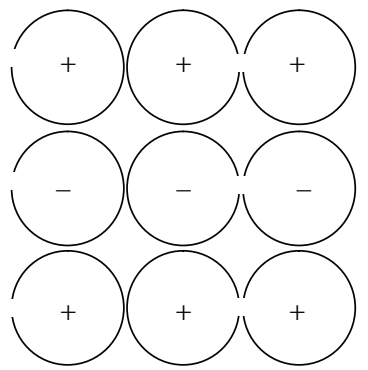

(e)

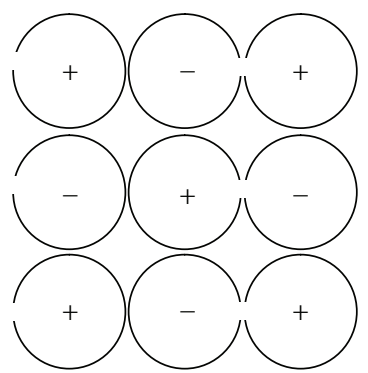

(f)

FIGURE 9: $3 \times 3$ lattices of fractional vortices each of charge \pm 3.5 embedded in a Fresnel lens. Simulated intensity distributions (a-c) and the corresponding charge distributions (d-f) are shown.

has no effect on the intensity. The field at the focal plane is proportional to the Fourier transform of the transmittance function given in (7). Intensity distribution at the observation plane is then given by

$$
I(r, \phi, f)=|E(r, \phi, f)|^{2} .
$$

Here, the intensity distribution refers to the far-field image of the object. We have carried our simulation studies to find the distribution of intensity in the focal plane.

\section{Experimental Setup}

A phase-only SLM can impose an azimuthal phase term directly on the incident beam converting it into a helical beam. The method of generating optical vortices using phase mask is more versatile because it allows precise and dynamic control over vortex parameters and possibility of generating specific patterns of optical vortices. The experimental arrangement is shown in Figure 2. A linearly polarized light from a He-Ne laser $(\lambda=632.8 \mathrm{~nm})$ is spatially filtered, expanded, and collimated to form a plane wave. The collimated beam of light is directed on the SLM (Holoeye LC-2002 with $832 \times 624$ pixels, pitch = $32 \mu \mathrm{m})$. By using properly oriented linear polarizer or by generating appropriate elliptically polarized light, one can modulate the phase while keeping the amplitude constant. We have used elliptically polarized light to achieve phaseonly modulation for the SLM using a combination of two polarizers and two quarter wave plates, as shown in Figure 2. This architecture consists of a combination of an input polarizer $\mathrm{P}$ and a quarter wave plate $\mathrm{QW}_{1}$ in front of the SLM and an output quarter wave plates $\mathrm{QW}_{2}$ and polarizer $\mathrm{P}_{2}$ behind the SLM. We adjust the polarizers and wave plates such that the SLM modulates only the phase, leaving the amplitude constant. A gray-scale phase mask corresponding to the transmittance of a lens, embedded with 
required vortex phase distributions, is displayed on SLM, and the corresponding intensity distribution is recorded on a CCD.

\section{Results and Discussion}

The Airy pattern at the focal plane of a lens is replaced by a doughnut pattern in a vortex lens. In a Fresnel vortex lens, the vortex phase is embedded at the extremum of the lens phase distribution that can produce a ring focus. By embedding multiple vortices in different locations in a Fresnel lens, vortex lattices can be realized at the focal plane [28]. Figure 3(a) shows the phase distribution of the modified Fresnel lens that can generate a $2 \times 2$ vortex lattice of four vortices with positive topological charge. Figures 3(b) and 3(c) depict the simulation and experimental results of the intensity pattern in the focal plane of the Fresnel lens embedded with $2 \times 2$ vortex lattice, as described above. The position and the charge of each of the vortices can be modified. Figure 4 shows one such configuration where two positive charge and two negative charge vortices are formed. The phase distributions are similar to that of a conventional Fresnel lens with branching of zones occurring at off-axis locations [29]. For positive (negative) vortex, the branch or fork occurs in anticlockwise (clockwise) sense as we move along the zone boundary. For a diverging Fresnel lens, the sense of fork formation is opposite to that of a positive lens. Similarly, vortex phase of fractional charge can also be embedded in a Fresnel lens. Figure 5 shows two positively charge fractional vortices of magnitude 3.5 , embedded at offaxis locations. In this case, apart from branching of zones at vortex points, there is an additional edge dislocation connecting the two vortices. In the intensity distribution near the focal plane, the two dark cores are connected by a curved dark line. The two dark cores and the connecting dark curved line are surrounded by bright intensity pattern. In Figure 6, oppositely charged fractional vortices are embedded in the phase distribution of Fresnel lens. The dark cores in this case are connected by the dark line that has different geometry. These intensity patterns are unique. Two fractional vortices are connected to each other, irrespective of the charge of the individual vortex. Figures 7 and 8 depict $2 \times 2$ arrays of vortices with fractional topological charges. The dark line that connects two vortices is decided by the manner, the edge dislocation connecting the two fractional vortices, appears in the phase distribution. If the edge dislocation line starting from one fractional vortex is not connected to another fractional vortex, it runs towards the boundary of the intensity pattern and terminates. Simulations are carried out to see the focal plane intensity distributions of such lenses. In all the Figures 3-8, it can be clearly seen that the experimental and simulation results match exactly. Figure 9 depicts vortex lattices of $3 \times 3$ arrays of the vortices with fractional topological charges. The charge distributions of the vortices in each of the lattices are different. Simulated intensity distributions at the focal plane corresponding to these lattices reveal that all the vortices are not connected by dark lines to form pairs. Only three pairs are connected, and in the remaining three fractional vortices, the dark lines from their cores are diverging outward.

\section{Conclusion}

Array of vortices of different charges are embedded in a wavefront with quadratic phase variation and the intensity distribution near the focal plane is studied. The intensity patterns, when fractional vortices are embedded in the Fresnel lens, possess unique interesting features. The intensity patterns produced by any two fractional charge vortices are connected by dark line, irrespective of the sign of the vortices. The geometry of this dark line is decided by the manner in which the edge dislocation line appears in the phase distribution of the Fresnel lens.

\section{References}

[1] J. F. Nye and M. V. Berry, "Dislocations in wave trains," Proceedings of the Royal Society A, vol. 336, pp. 165-190, 1974.

[2] W. M. Lee, X.-C. Yuan, and K. Dholakia, "Experimental observation of optical vortex evolution in a Gaussian beam with an embedded fractional phase step," Optics Communications, vol. 239, no. 1-3, pp. 129-135, 2004.

[3] M. V. Berry, "Optical vortices evolving from helicoidal integer and fractional phase steps," Journal of Optics A, vol. 6, no. 2, pp. 259-268, 2004.

[4] K. T. Gahagan and G. A. Swartzlander, "Optical vortex trapping of particles," Optics Letters, vol. 21, no. 11, pp. 827-829, 1996.

[5] A. Jesacher, S. Furhapter, S. Bernet, and M. Ritsch-Marte, "Spiral interferometery," Optics Letters, vol. 30, pp. 1953-1955, 2006.

[6] G. Gibson, J. Courtial, M. J. Padgett et al., "Free space information transfer using light beams carrying orbital angular momentum," Optics Express, vol. 12, no. 22, pp. 5448-5456, 2004.

[7] J. A. Davis, I. Moreno, and K. Crabtree, "Optical processing with vortex-producing lenses," Applied Optics, vol. 43, no. 6, pp. 1360-1367, 2004.

[8] P. Senthilkumaran, "Optical phase singularities in detection of laser beam collimation," Applied Optics, vol. 42, no. 31, pp. 6314-6320, 2003.

[9] G. A. Swartzlander Jr., "Peering into darkness with a vortex spatial filter," Optics Letters, vol. 26, no. 8, pp. 497-499, 2001.

[10] J. Leach, M. R. Dennis, J. Courtial, and M. J. Padgett, "Knotted threads of darkness," Nature, vol. 432, no. 7014, p. 165, 2004.

[11] D. P. Ghai, S. Vyas, P. Senthilkumaran, and R. S. Sirohi, "Vortex lattice generation using interferometric techniques based on lateral shearing," Optics Communications, vol. 282, no. 14, pp. 2692-2698, 2009.

[12] M. M. Burns, J. M. Fournier, and J. A. Golovchenko, "Optical matter: crystallization and binding in intense optical fields," Science, vol. 249, no. 4970, pp. 749-754, 1990.

[13] G. A. Swartzlander Jr. and C. T. Law, "Optical vortex solitons observed in Kerr nonlinear media," Physical Review Letters, vol. 69, no. 17, pp. 2503-2506, 1992.

[14] M. P. MacDonald, G. C. Spalding, and K. Dholakia, "Microfluidic sorting in an optical lattice," Nature, vol. 426, no. 6965, pp. 421-424, 2003. 
[15] J. Masajada, "Small-angle rotations measurement using optical vortex interferometer," Optics Communications, vol. 239, no. 4-6, pp. 373-381, 2004.

[16] K. Ladavac and D. G. Grier, "Micro-opto-mechanical pumps assembled and driven by holographic optical vortex arrays," Optics Express, vol. 12, no. 6, pp. 1144-1149, 2004.

[17] N. Chattrapiban, E. A. Rogers, I. V. Arakelyan, R. Roy, and W. T. Hill, "Laser beams with embedded vortices: tools for atom optics," Journal of the Optical Society of America B, vol. 23, no. 1, pp. 94-103, 2006.

[18] J. Masajada and B. Dubik, "Optical vortex generation by three plane wave interference," Optics Communications, vol. 198, no. 1-3, pp. 21-27, 2001.

[19] S. Vyas and P. Senthilkumaran, "Interferometric optical vortex array generator," Applied Optics, vol. 46, no. 15, pp. 2893 2898, 2007.

[20] J. Masajada, A. Popiołek-Masajada, and M. Leniec, "Creation of vortex lattices by a wavefront division," Optics Express, vol. 15, no. 8, pp. 5196-5207, 2007.

[21] G. X. Wei, L. L. Lu, and C. S. Guo, "Generation of optical vortex array using Talbot array illuminators," Optics Communications, vol. 282, pp. 2665-2669, 2009.

[22] C. S. Guo, Y. Zhang, Y. J. Han, J. P. Ding, and H. T. Wang, "Generation of optical vortices with arbitrary shape and array via helical phase spatial filtering," Optics Communications, vol. 259, no. 2, pp. 449-454, 2006.

[23] G. A. Swartzlander Jr., "Achromatic optical vortex lens," Optics Letters, vol. 31, no. 13, pp. 2042-2044, 2006.

[24] S. Vyas, R. Kumar Singh, and P. Senthilkumaran, "Fractional vortex lens," Optics and Laser Technology, vol. 42, no. 6, pp. 1150-1159, 2010.

[25] P. Senthilkumaran and F. Wyrowski, "Phase synthesis in waveoptical engineering: mapping and diffuser type approaches," Journal of Modern Optics, vol. 49, no. 11, pp. 1831-1850, 2002.

[26] P. Senthilkumaran, F. Wyrowski, and H. Schimmel, "Vortex Stagnation problem in iterative Fourier transform algorithms," Optics and Lasers in Engineering, vol. 43, no. 1, pp. 43-56, 2005.

[27] J. W. Goodman, Introduction to Fourier Optics, McGraw-Hill Companies, New York, NY, USA, 2nd edition, 1996.

[28] X. Gan, J. Zhao, S. Liu, and L. Fang, "Generation and motion control of optical multi-vortex," Chinese Optics Letters, vol. 7, no. 12, pp. 1142-1145, 2009.

[29] F. S. Roux, "Diffractive lens with a null in the center of its focal point," Applied Optics, vol. 32, no. 22, pp. 4191-4192, 1993. 

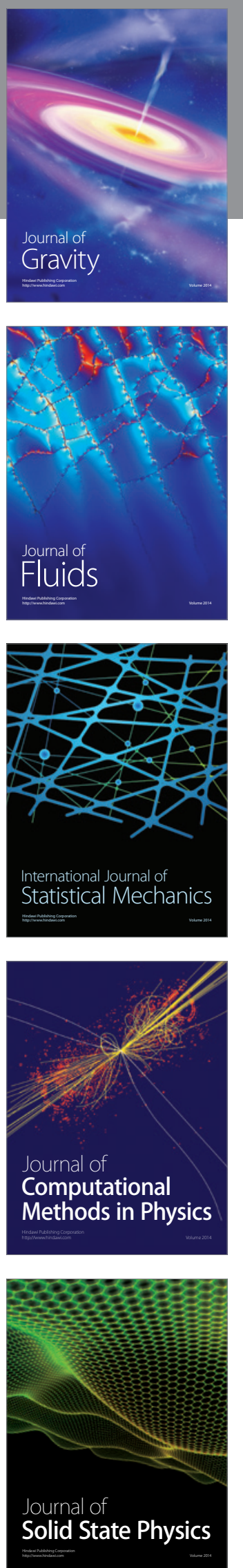

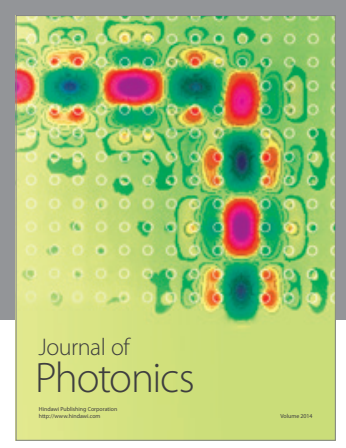

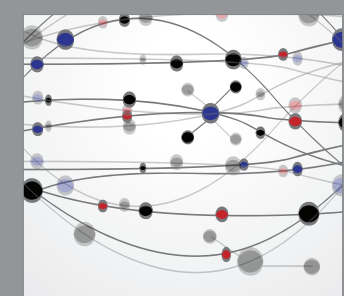

The Scientific World Journal
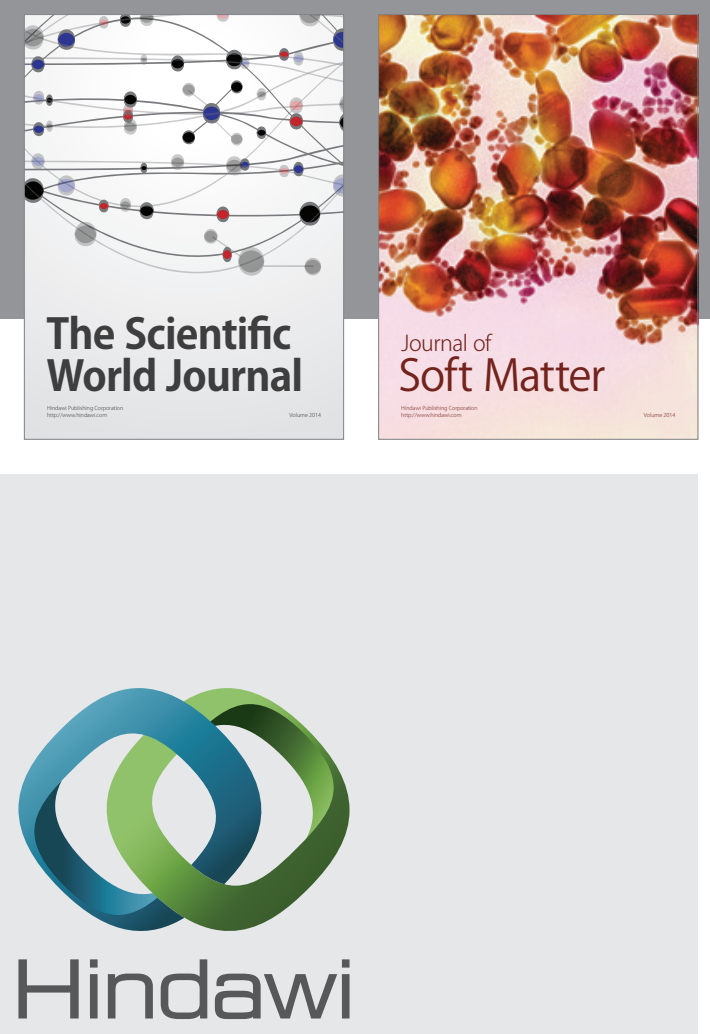

Submit your manuscripts at

http://www.hindawi.com
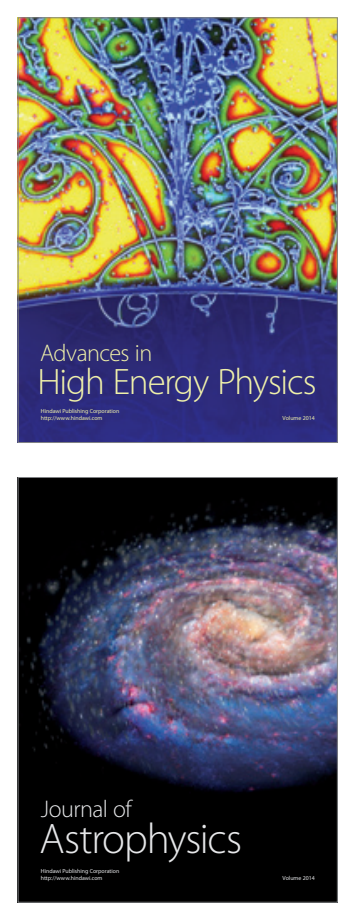
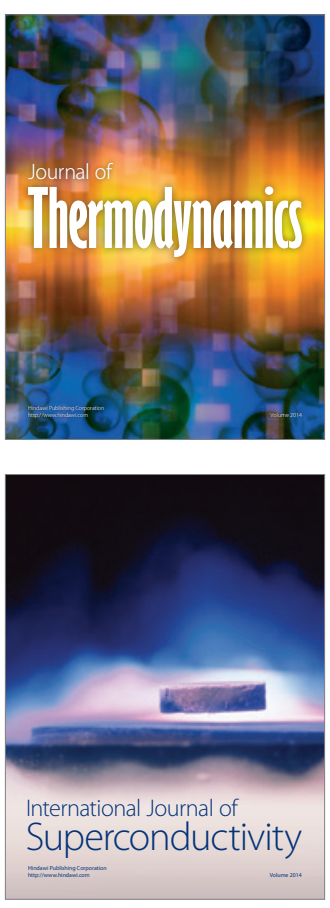
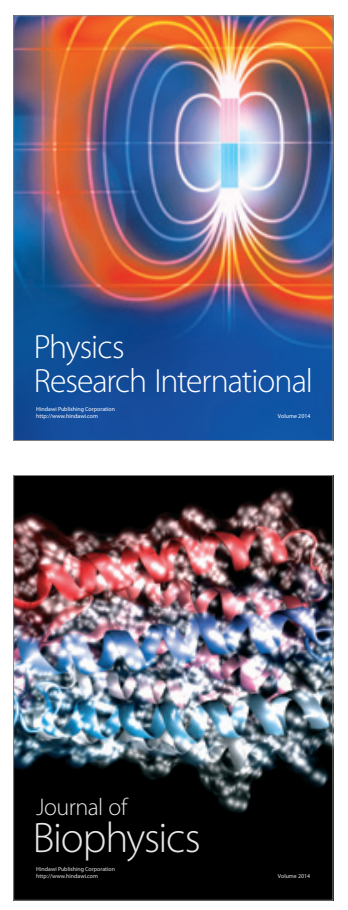
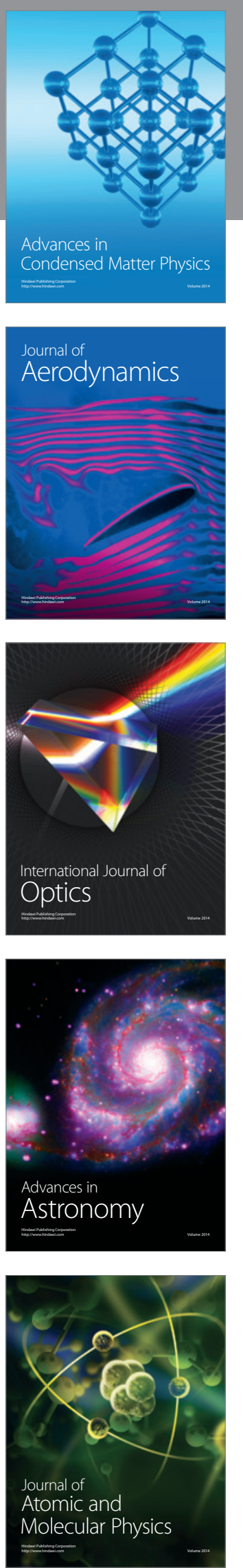